\title{
A SCANNING ELECTRON MICROSCOPIC STUDY OF HYPERCEMENTOSIS
}

\author{
Bethânia Camargo PINHEIROํ, Tiago Novaes PINHEIRO¹, Ana Lúcia Alvarez CAPELOZZA², Alberto CONSOLARO³
}

1- DDS, Master's degree in Oral Pathology, Bauru School of Dentistry, University of São Paulo, Bauru, SP, Brazil.

2- DDS, MSc, PhD, Associate Professor, Discipline of Radiology, Department of Stomatology, Bauru School of Dentistry, University of São Paulo, Bauru, SP, Brazil.

3- DDS, MSc, PhD, Full Professor, Discipline of Oral Pathology, Department of Stomatology, Bauru School of Dentistry, University of São Paulo, Bauru, SP, Brazil.

Corresponding address: Dra. Bethânia Camargo Pinheiro - Rua Garcia Braga, 126, Centro, 18940-000, São Pedro do Turvo, SP, Brasil.

Phone: +55-14-3377-1190 - e-mail: bethaniacp@hotmail.com

Received: February 22, 2008 - Modification: April 18, 2008 - Accepted: May 08, 2008

\begin{abstract}
$T_{\text {he }}$ L he purpose or this study was to evaluate norphologieal characteristics of teeth with hypercementosis that are relevant to endodontic practice. Twenty-eight extracted teeth with hypercementosis had their root apexes analyzed by scanning electron microscopy (SEM). The teeth were divided according to tooth groups and type of hypercementosis. The following aspects were examined under SEM: the contour and regularity of the root surface; presence of resorption; presence and number of apical foramina, and the diameter of the main foramen. The progression of club shape hypercementosis was directly associated with the presence of foramina and apical foramen obstruction. Cases of focal hypercementosis presented foramina on the surface, even when sidelong located in the root. Circular cementum hyperplasia form was present in 2 out of 3 residual roots, which was the highest proportion among the tooth types. The detection of a large number of foramina in the apical third of teeth with hypercementosis or even the possible existence of apical foramen obliteration contributes to understand the difficulties faced during endodontic treatment of these cases.
\end{abstract}

Key Words: Hypercementosis. Root canal. Morphology. Endodontics.

\section{INTRODUCTION}

Hypercementosis is characterized by cementum formation beyond the physiologic limits of the tooth. This excessive amount of cementum might lead to an abnormal thickness of the apex that becomes round-shaped and/or with the root appearance altered macroscopically ${ }^{6}$.

The incidence of hypercementosis by race or population group has not yet been established. Grzesik, et al. ${ }^{8}$ studied the frequency of this phenomenon in 137 individuals and observed an average of 3.8 teeth with hypercementosis per individual aged approximately 47 years, and the premolars were the most affected teeth ${ }^{8}$. Other studies reported root hypercementosis in $84 \%$ out of 104 Barbados slave skeletal collection from the 17 th to 19 th centuries 5 . Root hypercementosis was also diagnosed in 10 out of 54 specimens gathered from prehistoric coastal populations of Texas, USA ${ }^{4}$.

The origin of hypercementosis is attributed to conditions such as functional stress due to occlusion forces ${ }^{4,5,7,19}$, continuous dental eruption ${ }^{2,3,9,12}$, incorporation of periodontal cementicles during physiologic cementum deposition ${ }^{6}$, reactionary deposition in response to periapical inflammatory processes ${ }^{18}$, systemic factors such as atherosclerosis, acromegaly, deforming arthritis, hypertrophic arthritis, thyroid diseases and Paget's disease ${ }^{1,10,13,17}$. Figure 1 shows the alterations in dental root morphology due to hypercementosis.

Radiographically, hypercementosis does not alter the biologic width between the root surface, the periodontal ligament, and the alveolar bone. Although hypercementosis can be identified radiographically, it is not possible to estimate the amount of extra cementum present in the affected root because dentin and cementum have the same radiodensity ${ }^{7,14}$.

Microscopic studies about hypercementosis have reported thick layers of cementum characterized by deposition of symmetric, highly basophilic lines parallel to dentin surface ${ }^{6,7}$. Occasionally, atypical cementum depositions can be identified in focal areas as external cementum projections. The presence of blood vessels and nervous filaments associated to irregular apical cementum deposition could contribute to the formation of multiple foramina during cementum deposition, due to 
hypercementosis, originating the apical deltas ${ }^{19}$.

The lack of recent studies referring to this subject, added to the potential clinical implications stimulate the evaluation of morphologic characteristics of the apical root third and root canal in teeth with hypercementosis. The purpose of
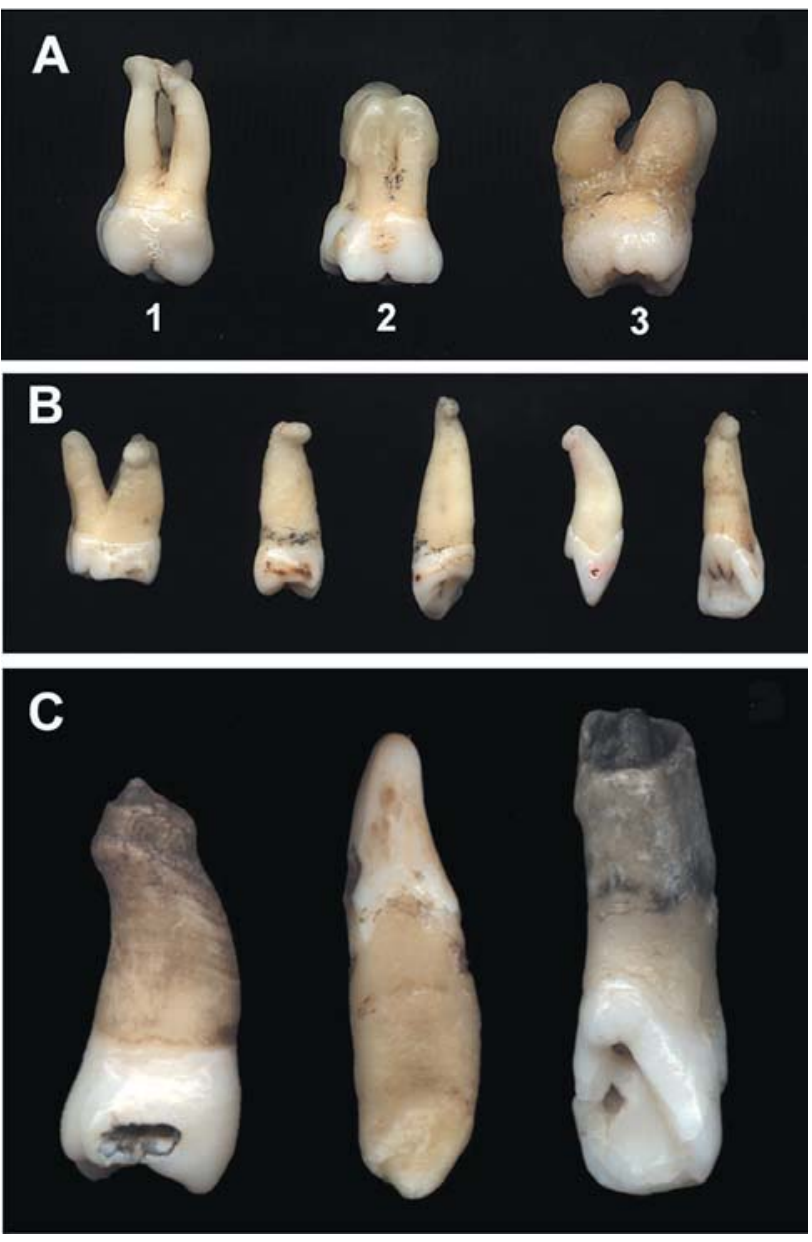

FIGURE 1- Macroscopic aspects of teeth with different types of hypercementosis. (A) Club shape hypercementosis: mild

(1), moderate (2) and severe (3). (B) Focal hypercementosis.

(C) Circular cementum hyperplasia hypercementosis this study was to examining by scanning electron microscopy (SEM) the external anatomy of the apical third of teeth with hypercementosis, assessing the existence of any relationship between hypercementosis and the diameter of the main foramen and the presence of apical foramina. These morphological features might implicate specific aspects on endodontic therapeutic techniques.

\section{MATERIAL AND METHODS}

Twenty-eight teeth with hypercementosis were selected from the archives of the Discipline of Oral Pathology at the Bauru School of Dentistry, SP, Brazil. Hypercementosis distribution was classified in the present study as: club shape, focal or circular cementum hyperplasia $(\mathrm{CCH})$. The longitudinal extension of club shape hypercementosis in the root thirds was graded as mild, moderate and severe. Regarding the tooth type, the sample comprised: 8 maxillary molars, 6 mandibular molars, 6 maxillary premolars, 1 mandibular premolar, 4 canines and 3 residual roots. Sample distribution according to hypercementosis type, tooth type and number of evaluated roots is displayed in Table 1.

The teeth had the crown and part of the roots sectioned transversally with carborundum disk and discarded, leaving 5-mm-long apical root segments. This procedure was necessary in order to fit the specimen in a metallic base used for SEM analysis. A total of 41 specimens were obtained from 46 roots.

The specimens were dehydrated by immersion in an ascending ethanol series (70, 80, 90 and 100\%), sputtercoated with gold and examined with a scanning electron microscope (Zeiss DSM-940A, Oberkochen, Germany) operating at $15 \mathrm{kV}$. The following aspects were examined in the SEM analysis: the contour and regularity of the root surface; presence of resorption; presence and the number of root foramina, and the diameter of the main foramen.

TABLE 1- Distribution of the 28 teeth according to the tooth group, type of hypercementosis and number of examined roots

Hypercementosis type

\begin{tabular}{|c|c|c|c|c|c|c|c|c|}
\hline & \multicolumn{3}{|c|}{ Club shape } & \multicolumn{3}{|c|}{ Focal } & \multicolumn{2}{|c|}{$\mathrm{CCH}$} \\
\hline & discrete & $\begin{array}{c}\text { Teeth } \\
\text { moderate }\end{array}$ & severe & Roots & Teeth & Roots & Teeth & Roots \\
\hline Maxillary molars & 2 & 1 & 2 & 15 & 2 & 2 & 1 & 3 \\
\hline Mandibular molars & 2 & 1 & & 6 & 1 & 1 & 2 & 4 \\
\hline Maxillary premolars & 1 & 1 & 2 & 5 & 1 & 1 & 1 & 1 \\
\hline Mandibular premolars & - & 1 & - & 1 & - & - & - & - \\
\hline Maxillary canines & - & 1 & 1 & 2 & 2 & 2 & - & - \\
\hline Residual roots & - & - & - & - & 1 & 1 & 2 & 2 \\
\hline Total & 5 & 5 & 5 & $29^{*}$ & 7 & $7^{*}$ & 6 & $10^{*}$ \\
\hline
\end{tabular}

* A total of 41 specimens were obtained from a total of 46 roots. 


\section{RESULTS}

\section{Club Shape Hypercementosis}

Out of 12 specimen with mild club shape hypercementosis $7(58.3 \%)$ exhibited the apical foramen, 6 $(50 \%)$ foramina and $3(25 \%)$ had the obliteration of the main foramen. Four 4 specimens (33.3\%) presented more than one of the studied aspects. Regarding the main foramen diameter, a 150-300 $\mu \mathrm{m}$ range was found. The apical surface of the teeth with this type of hypercementosis showed sometimes a regular or an irregular aspect.

It was not possible to observe the apical foramen in only 1 specimen of teeth with moderate club shape hypercementosis. The foramen diameter varied between 150 and $450 \mu \mathrm{m}$. A larger number of roots presented irregular surfaces due to apical resorption as well as the union of the roots by the cementum and the presence of an apical main foramen and foramina (Figure 2).

Seven specimens presented severe club shape hypercementosis. Only 3 cases (42.8\%) showed apical main foramen and apical foramina. The main foramen diameter varied between 100 and $300 \mu \mathrm{m}$. Figure 3 shows the presence of tiny foramina at the apex of the mesiobuccal root of a maxillary molar. A mineralized structure partially obliterating the apical foramina was observed in 1 specimen.

\section{CCH Hypercementosis}

The CCH type hypercementosis presented depressions close to the apical foramen and thick cementum grossly deposited on the lateral surface of the root. Figure 4 presents buccal roots of a maxillary molar with depressions close to the apical foramen and a thick cementum layer on the lateral root surface. The average diameter of the main apical foramen was $325 \mu \mathrm{m}$, and only 3 specimens $(42.85 \%)$ presented foramina. A mandibular molar and a residual root also presented irregularities due to the presence of areas of resorption.

\section{Focal hypercementosis}

Regular foramina were observed in the teeth with focal hypercementosis. The maxillary canines showed foramina in areas of irregular cementum deposition (Figure 5). Data referring to the main apical foramen and foramina are displayed presented in Table 2.

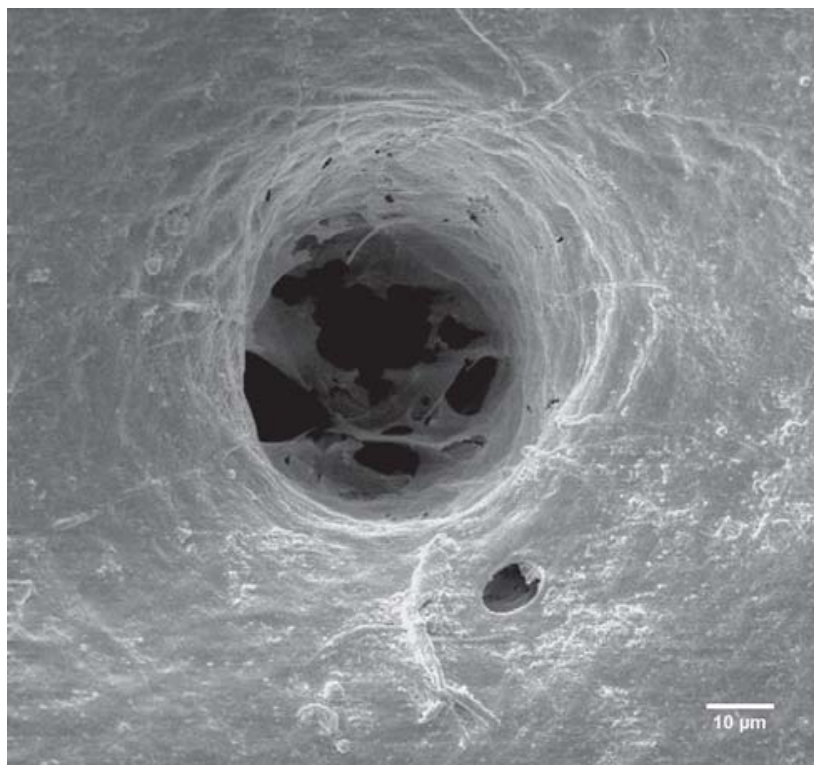

FIGURE 3- Severe club shape hypercementosis. Root apex of a maxillary molar, showing the presence of 2 apical foramina. Original magnification: $\times 1,000$
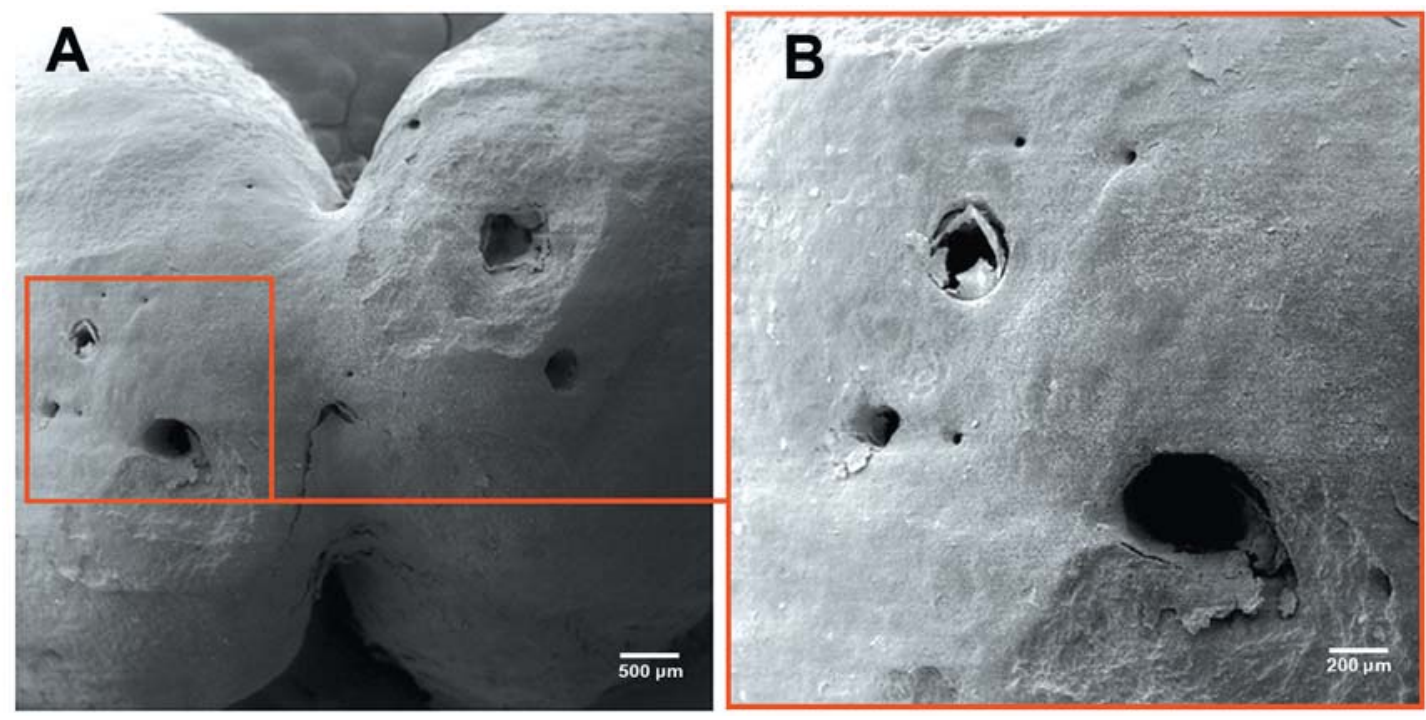

FIGURE 2- Moderate club shape hypercementosis. (A) Mesial and distal roots of a mandibular molar. (B) Greater magnification of the apical foramen and foramina. Irregular root resorption areas are observed close to the apical foramen and foramina. Original magnifications: $(A) \times 20,(B) \times 50$ 


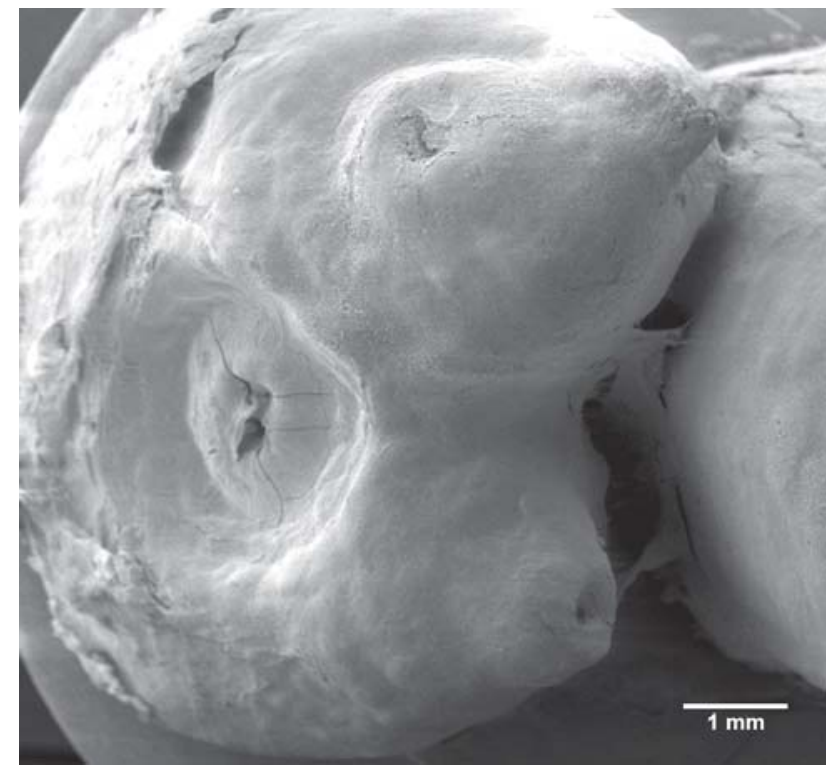

FIGURE 4- Circular cementum hyperplasia hypercementosis. Original magnification: $\times 15$

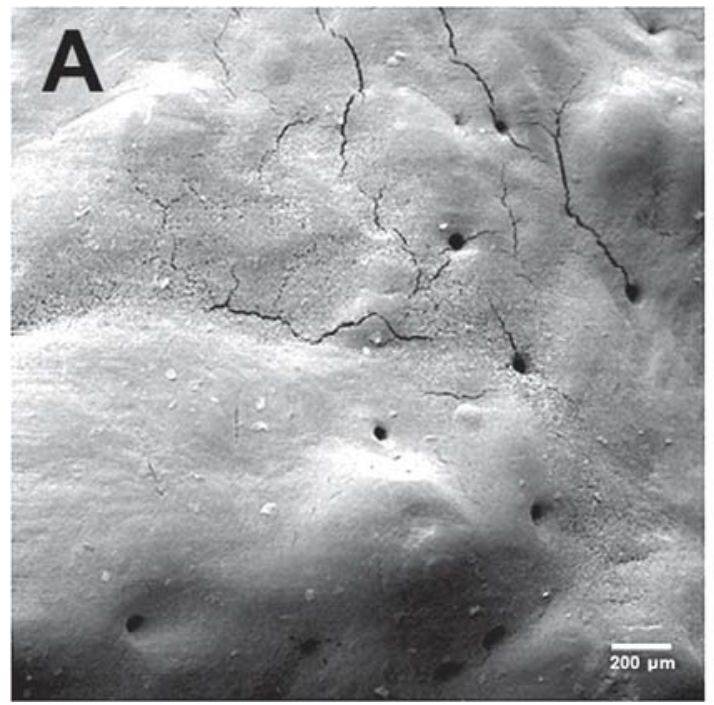

\section{DISCUSSION}

Although there are not many studies regarding this subject, hypercementosis can be considered as a common finding in endodontic daily practice. The impact of hypercementosis on endodontic prognosis and its direct influence on therapeutic procedures still unclear ${ }^{20}$. Whether to keep instrumentation and root canal filling above or beyond the cementum-dentin-canal junction in teeth with hypercementosis is a difficult clinical choice. The apical limits for root canal preparation depend on different reference landmarks ${ }^{16}$, and hypercementosis might impair such decisions.

In the present study, SEM analysis of the root apex of permanent teeth with hypercementosis provided a rapid evaluation of the structure with identification of the main foramen and apical foramina, and determination of their dimensions. Moreover how and where the apical foramen and accessory foramina are displaced due to hypercementosis, and possible variations related to its morphotypes were observed. Therefore, knowing the apical surface of teeth with hypercementosis is important for endodontic treatment planning.

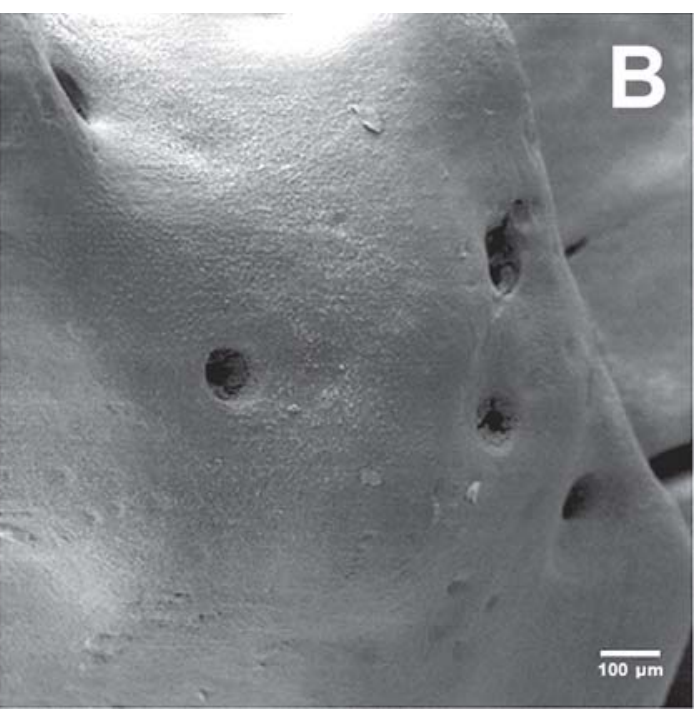

FIGURE 5- Focal hypercementosis. (A) Morphologic aspects of the foramina located in the distobuccal root of a maxillary molar. (B) Openings of the foramina at the middle third at the mesiobuccal surface of a maxillary canine. Original magnifications: (A) $\times 100,(B) \times 50$

TABLE 2- Apical main foramen and foramina observed in the 41 examined specimens

\begin{tabular}{|c|c|c|c|c|c|c|c|c|}
\hline \multicolumn{2}{|c|}{ Hypercementosis } & \multirow[t]{2}{*}{ Specimens } & \multicolumn{2}{|c|}{ Main foramen } & \multicolumn{2}{|c|}{ Foramina } & \multicolumn{2}{|c|}{ Obliteration } \\
\hline & & & $\mathbf{n}$ & $\%$ & & $\%$ & n & $\%$ \\
\hline & mild & 12 & 7 & 58.3 & 6 & 50.0 & 3 & 25.0 \\
\hline \multirow[t]{2}{*}{ Club shape } & moderate & 7 & 6 & 85.7 & 6 & 85.7 & 0 & 0 \\
\hline & severe & 7 & 3 & 42.8 & 3 & 42.8 & 4 & 57.1 \\
\hline $\mathrm{CCH}$ & & 7 & 6 & 85.7 & 3 & 42.8 & 1 & 14.3 \\
\hline Focal & & 8 & 0 & 0 & 6 & 75.0 & 0 & 0 \\
\hline Total & & 41 & 22 & 53.6 & 24 & 58.5 & 8 & 19.5 \\
\hline
\end{tabular}


The diameter of the main apical foramen ranged in our study from 100 to $500 \mu \mathrm{m}$. Openings with diameters inferior to $100 \mu \mathrm{m}$ were considered as apical foramina ${ }^{15}$. The presence of apical foramina in areas with hypercementosis would indicate that cementum deposition occurred respecting the neurovascular supply ${ }^{18}$. In the present study, the observation of apical foramina in 24 specimens supports this idea. The absence of cementum deposition obliterating those foramina may be related by cytokines and growth factors released by endothelial cells and nervous branches ${ }^{8}$. Partial or total obliteration of the apical foramina found in the present study could be a reactive response to the cause of the tooth extraction ${ }^{8}$.

Most root apexes of teeth with mild club shape hypercementosis did not present surface irregularities and cementum resorption, though presenting a large number of apical foramina. Moderate club shape hypercementosis showed irregular areas and apical foramina. The severe club shape hypercementosis presented a decrease in the number and sometimes obliteration of the apical foramen.

The present study found that the circular cementum hyperplasia type of hypercementosis was not associated with apical resorption. Unless proven otherwise, reactive mechanisms due to chronic periapical lesions can be involved in the process. This type of hypercementosis might represent additional anatomic niches to bacterial colonization during root canal infection, which contributes to the presence of refractory lesions ${ }^{21}$.

The focal type of hypercementosis presents foramina on its surface, even when sidelong located on the roots. This observation warns for the development of endo-perio lesions if these openings are be reached by bacteria and its toxic products $^{11,21}$

\section{CONCLUSIONS}

The detection of a large number of foramina in the apical third of teeth with hypercementosis or even the possible existence of apical foramen obliteration contributes to understand the difficulties faced during endodontic treatment of these cases. Constant research seeking the development of techniques and materials that provide a correct instrumentation and filling of these teeth should be encouraged.

\section{REFERENCES}

1- Aldred MJ, Cooke BED. Paget's disease of bone with involvement of the dental pulp. J Oral Pathol Med. 1989;18:184-5.

2- Azaz B, Ulmansky M, Moshev R, Sela J. Correlation between age and thickness of cementum in impacted teeth. Oral Surg Oral Med Oral Pathol. 1974;38:691-4

3- Azaz B, Michaeli Y, Nitzan D. Aging of tissues of the roots of nonfunctional human teeth. Oral Surg. 1977;43:572-8.
4- Comuzzie AG, Steele DG. Enlarged occlusal surfaces on first molars due to severe attrition and hypercementosis: examples from prehistoric coastal populations of Texas. Am J Phys Anthropol. 1989;78:9-15.

5- Corruccini RS, Jacobi KP, Handler JS, Aufderheide AC. Implications of tooth root hypercementosis in a Barbados slave skeletal collection. Am J Phys Anthropol. 1987;74:179-84.

6- Dewey KW. Normal and pathological cementum formation. Dent Cosmos. 1926;68:560-85

7- Gardner BS, Goldstein H. The significance of hypercementosis. Dent Cosmos. 1931;73:1065-9.

8- Grzesik WJ, Narayanan AS. Cementum and periodontal wound healing and regeneration. Crit Rev Oral Biol Med. 2002;13:474-84.

9- Hopewell-Smith A. Concerning human cementum. J Dent Res. 1920;2:59-76

10- Israel H. Early hypercementosis and arrested dental eruption: heritable multiple ankylodontia. J Craniofac Genet Dev Biol. 1984;4:243-6.

11- Kerr DA. The cementum: its role in periodontal health and disease. J Periodontol. 1961;32:183-9.

12- Kronfeld R. The biology of cementum. J Am Dent Assoc. 1938;25:1451-61.

13- Leider AS, Garbarino VE. Generalized hypercementosis. Oral Surg Oral Med Oral Pathol. 1987;63:375-80.

14- Monahan R. Periapical and localized radiopacities. Dent Clin North Am. 1994;38:113-36

15- Morfis A, Sylaras SN, Georgopoulou M, Kernani M, Prountzos F. Study of the apices of human permanent teeth with the use of a scanning electron microscope. Oral Surg Oral Med Oral Pathol. 1994;77:172-6.

16- Ponce EH, Fernández JAV. The Cemento-dentino-canal junction, the apical foramen, and the apical constriction: evaluation by optical microscopy. J Endod. 2003;29:214-9.

17- Soni NN. A microradiographic and polarized light study of cementum in Paget's disease. J Oral Med. 1969;24:27-30.

18- Thoma KH, Goldman HM. The pathology of dental cementum. J Am Dent Assoc. 1939;26:1943-53

19- Thomas NG. Formation of the apices of teeth. J Am Dent Assoc. 1921;8:11-7.

20- Weinberger A. Clinical significance of hypercmentosis. Oral Surg Oral Med Oral Pathol. 1954;7:79-87.

21- Zehnder M, Gold SI, Hasselgren G. Pathologic interactions in pulpal and periodontal tissues. J Clin Periodontol. 2002;29:663-71. 